\title{
Probing the Conduction Channels of Gold Atomic-Size Contacts: Proximity Effect and Multiple Andreev Reflections
}

\author{
E. Scheer ${ }^{1}$, W. Belzig 2 , Y. Naveh ${ }^{3}$, and C. Urbina ${ }^{4}$ \\ 1 Fachbereich Physik, Universität Konstanz, 78457 Konstanz, Germany \\ 2 Departement Physik, Universität Basel, 4056 Basel, Switzerland \\ 3 Department of Physics, SUNY at Stony Brook, New York 11794, USA \\ 4 SPEC, CEA-Saclay, 91191 Gif-sur-Yvette Cedex, France
}

\begin{abstract}
We have investigated the electronic transport properties of Au quantum point contacts fabricated by the mechanically controllable breakjunction technique using non-linearities in the current-voltage characteristics induced by the proximity effect (PE) of superconducting aluminum electrodes. The PE shows up also in an hysteretic transition to the normal state when applying an external magnetic field. We find that the very smallest contact formed by a single atom between the electrodes contributes one channel to the transport as predicted by different theoretical models. The analysis of the transport channels of retraceable opening and closing procedures gives additional evidence that the transport channels are determined by the properties of the central atom.
\end{abstract}

Since the development of the scanning tunnelling microscope (STM) [1] it is not only possible to see, but also to manipulate and to measure the transport properties of individual atoms on surfaces [2]. By energy dependent measurements of the differential conductance a certain chemical information can be achieved [3]. The challenging aim of building up electronic circuits atom by atom with tailor-made properties, however, would require the detailed knowledge of the relation between the physical and chemical properties of the respective atoms and their conduction properties, a problem which has been addressed by different methods during the last years [4]. The most simple system for all investigations - including the present - is a one-atom contact between two metallic banks of the same element.

Electrical transport through such contacts is suitably described by the Landauer formalism, which treats it as a wave scattering problem. The transport properties of the contact connected to the leads are described by a set of transmission coefficients $\left\{T_{\mathrm{n}}\right\}$ with $T_{\mathrm{n}} \leq 1$. E.g. the conductance $G$ of a contact is $G=G_{0} \sum_{\mathrm{n}} T_{\mathrm{n}}$. Here, $G_{0}=2 e^{2} / h$ is the conductance quantum [5]. Since in few-atom metallic point contacts the structure size is of the same order as the Fermi wavelength, such a contact has only a small number $N$ of channels. If the set $\left\{T_{\mathrm{n}}\right\}$ is known, many further transport properties, as e.g. shot noise, thermopower or superconducting properties of the contact can be deduced [6]. 
In order to test basic concepts of the quantum mechanical transport theory it is of particular interest to study metallic systems transmitting only one single channel with adjustable transmission coefficient. According to a quantum chemical model by Cuevas, Levy Yeyati and Martín-Rodero $[7,8]$ the transmission coefficients of single-atom contacts are a function of the chemical properties of the metal and the atomic arrangement of the region around the central atom. Within their model only single atom contacts of monovalent metals as e.g. the alkali or noble metals should transmit one single channel. In particular for $\mathrm{Au}$ it has been predicted that the transmission coefficient of this single channel can achieve, in a perfectly ordered geometry of the central atom and its neighbors, a nearly saturated value of $T>0.99$ [8]. We present here results of an experiment which allows to determine the transmission coefficients of this model substance with the help of proximity superconductivity induced by aluminum electrodes close to the contact region. We discuss the properties of the $\mathrm{PE}$ in a perpendicular magnetic field.

It has been shown [9] that the set $\left\{T_{\mathrm{n}}\right\}$ of atomic-size constrictions is amenable to measurement using the strong non-linearities in the currentvoltage $(I V)$ characteristics of superconducting atomic contacts due to multiple Andreev reflections (MAR) [10]. Fig. 1 a) shows the numerical predictions for a single channel with transmission $T[11,12,13,14]$. The $i(V, T)$ curves present a series of sharp current steps at voltage values $\mathrm{eV}=2 \Delta / \mathrm{m}$, where $m$ is a positive integer and $\Delta$ is the superconducting gap. Each one of these steps corresponds to an additional microscopic process of charge transfer as depicted in Fig. 1 b)-d). For example, the well-known non-linearity at $e V=2 \Delta$ arises when one electronic charge $(m=1)$ is transferred. The order $m=2,3, \ldots$ of a step corresponds to the number of electronic charges transferred in the underlying MAR process. Energy conservation imposes a threshold $e V \geq 2 \Delta / m$ for the process of order $m$. For low transmission, the contribution to the current arising from the process of order $m$ scales as $T^{m}$. The analysis of the transmission coefficients is performed by decomposing the experimental $I V \mathrm{~s}$ of a particular contact into contributions of $N$ independent terms with the $\left\{T_{n}\right\}$ (where $n=1, \ldots, N$ ) as fitting parameters: $I(V)=\sum_{n=1}^{N} i\left(V, T_{n}\right)$. The important result of this analysis is that single-atom contacts of multivalent metals like $\mathrm{Al}, \mathrm{Pb}$ or $\mathrm{Nb}$ always transmit more than one channel. The transmission coefficients of the channels depend sensitively on the exact atomic arrangement of the contact $[9,15]$. Since $\mathrm{Au}$ is not superconducting, the described method of determining conduction channels is not directly applicable. However, due to the so-called proximity effect (PE) $[16,17]$, a finite piece of a non-superconducting metal in good contact with a superconducting metal adopts certain superconducting properties, e.g. it develops a "minigap" $E_{\mathrm{g}}$ in the quasiparticle excitation spectrum. As will be explained below, the appearance of the minigap is the basic property for determining the channel ensemble. 

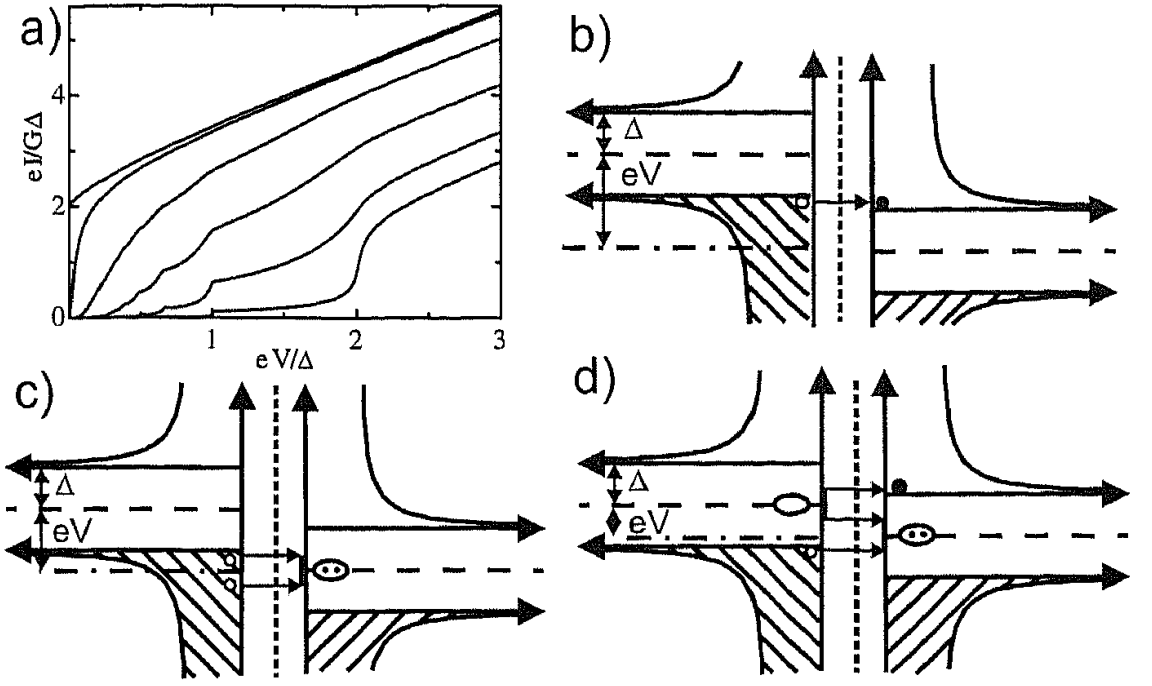

Fig. 1. a): Theoretical current voltage characteristics for a single-channel superconducting contact for different values of the transmission $T$ (from bottom to top: $0.1,0.4,0.7,0.9,0.99,1$ after [13]). b)-d): Representation of the transport processes of MAR at zero temperature: The quasiparticle (qp) densities of states for the left and right electrode forming the contact in a modified semiconductor model are shown. Qp's are represented as open circles when removed from an electrode and closed circles when added to an electrode. In addition the Cooper pairs (Cp's) are shown as ellipses on the Fermi levels (empty ellipses when a Cp is broken, ellipses filled with two circles when a $\mathrm{Cp}$ is formed) Energy conservation requires transport processes which are horizontal. The contact is represented by the vertical dotted line in the middle. Due to the contact with finite resistance it is possible to apply a voltage $\mathrm{eV}$ between the left and the right electrode, measured between the Fermi energies (dashed horizontal lines) of the electrodes. In the examples shown in b)-d) a positive voltage is applied to the right electrodes such that the charges are transported form the left to the right electrode. b) When the applied voltage exceeds $2 \Delta / e$, transport of a single qp is possible, corresponding to the MAR process with $m=1$. c) For voltages $V \geq 2 \Delta / 2 e$ the simultaneous transport of two qp's, forming a $\mathrm{Cp}$ in the right electrode also contributes to the current $(m=2)$. d) For $V \geq 2 \Delta / 3 e$ three qp's are transferred in a process where in the left electrode a Cp is broken, a new $\mathrm{Cp}$ is formed in the right electrode and a qp moved from the occupied states at the left electrode to the empty states in the right electrode

Experimentally, stable atomic-size contacts can be achieved with different techniques including STM $[18,19]$ and mechanically controllable breakjunctions (MCB) [20]. Using such methods it has been shown that smallest stable contacts of gold have most often a conductance close to $G_{0}$. We have produced micro-fabricated breakjunctions [21] of $\mathrm{Al}$, where the center part of the constriction consists of $\mathrm{Au}$. Using shadow evaporation through a suspended mask we evaporate perpendicular to the substrate surface two Al electrodes separated by a gap of width $2 L_{N}$. Without breaking the vacuum, two $\mathrm{Au}$ layers of thickness $d_{a u} / 2$ are evaporated at two different angles $\pm 10^{\circ}$ in order to fill the gap and to form a continuous film (see Fig. 2). For sample parameters see Table 1. After lifting off the mask the underlying sacrificial 


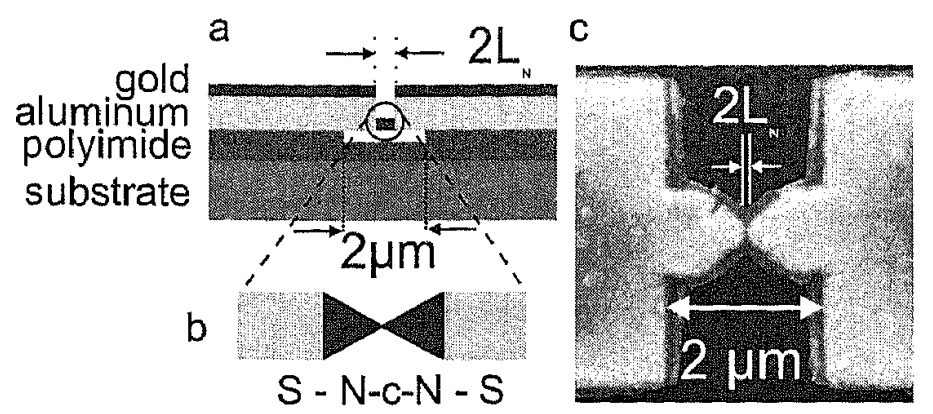

Fig. 2. a) Schematic side view of the samples. The metallic thin films are evaporated on top of a flexible substrate covered by a polyimide sacrificial layer. The latter is etched away to suspend the central bridge that connects the two large $\mathrm{Al}$ electrodes trough a small Au constriction. b) Schematic top view of the central part of the resulting system. Two nominally identical NS structures coupled through a constriction which can be controlled in-situ down to the atomic size. c) SEM micrograph of sample No. 1 (top view). The $\mathrm{Al}$ electrodes are the brightest regions. The narrow and dark skirt around them corresponds to the gold layer, through which the contact is established

Table 1. Sample number, thickness of the Al [Au] layer $d_{A l[A u]}$, elastic mean free path of the Au layer $l_{A u}$, mini-gap $E_{g}$ [superconducting gap $\Delta$ for sample No. 4], critical temperature $\Theta_{c}$, critical field $B_{c}$, spacing between Al electrodes as deduced by best fit to the theory of Ref. [17] $\left(L_{N} / \xi_{S}\right)_{f i t}$ (assuming $\xi_{S}=280 \mathrm{~nm}$ ) and as estimated from the micrographs $\left(L_{N} / \xi_{S}\right)_{e x p}$, and best fit value $\Gamma_{f i t}$ and estimated value $\Gamma_{\exp }$ of the mismatch parameter. For sample No. 3 this detailed analysis has not been performed.

\begin{tabular}{ccccccccccc} 
No. & $d_{A l}$ & $d_{A u}$ & $l_{A u}$ & $E_{g}[\Delta]$ & $\Theta_{c}$ & \multicolumn{3}{c}{$B_{c}$} & $\left(L_{N} / \xi_{S}\right)$ & \multicolumn{2}{c}{$\Gamma$} \\
& $(\mathrm{nm})$ & $(\mathrm{nm})$ & $(\mathrm{nm})$ & $(\mu \mathrm{eV})$ & $\mathrm{K}$ & $\mathrm{mT}$ & fit & $\exp$ & fit & $\exp$ \\
\hline 1 & 300 & 20 & 45 & 160 & 1.21 & 6.35 & 0.16 & 0.1 & 0.6 & 0.54 \\
2 & 400 & 30 & 25 & 140 & 1.21 & 5.05 & 0.8 & 0.4 & 0.2 & 0.4 \\
3 & 400 & 40 & 35 & 125 & 1.05 & 5.02 & & & & \\
4 & 150 & - & - & 180 & 1.21 & 10.2 & - & - & - & -
\end{tabular}

polyimide layer is partially dry etched to form a free-standing nanobridge over $2 \mu \mathrm{m}$ length. For comparison we also produced several breakjunctions made of an continuous Al film of thickness $150 \mathrm{~nm}$ to $200 \mathrm{~nm}$.

The bridge is broken at the constriction at very low temperatures $\Theta<1 \mathrm{~K}$ and under cryogenic vacuum conditions by controlled bending of the elastic substrate mounted on a three-point bending mechanism. Details of the sample preparation and measuring setup are given in $[9,22,23]$. As found in previous experiments [20] the conductance $G$ decreases in steps of the order of $G_{0}$, with smaller steps within a plateau (see Ref. $[9,15]$ ). The last contact before the wire breaks (the breaking is indicated by the onset of exponential decrease of $G$ with distance of the electrodes) is usually below $1 G_{0}$. We attribute this observation to the disorder in the contact region induced during the ion etching process. 
From experiments on pure Al samples [9] we estimate the superconducting gap $\Delta=180 \mu \mathrm{eV}$, the diffusion constant $D=v_{\mathrm{F}} l_{\mathrm{Al}} / 3=0.042 \mathrm{~m}^{2} / \mathrm{s}$ and the coherence length $\xi=(\hbar D / 2 \Delta)^{1 / 2}=280 \mathrm{~nm}$. From the residual resistance ratio $\mathrm{RRR}=R(300 \mathrm{~K}) / R(4 \mathrm{~K})$ of the Au layers we deduce an elastic mean free path of $l_{\mathrm{A} u} \approx 25-45 \mathrm{~nm}$.

For recording current-voltage characteristics $(I V \mathrm{~s})$ the motion of the bending mechanism can be stopped at any conductance value $G$. Fig. 3 shows the $I V \mathrm{~s}$ of two Al-Au-Al samples and an $\mathrm{Al}$ sample obtained in the tunnel regime at a temperature of $50 \mathrm{mK}$.

A clear minigap $E_{\mathrm{g}}<\Delta$ is observed for both samples and a maximum of the current slightly above the minigap. This maximum is a consequence of the deviation of the density of states (DOS) $\rho(E)$ from the BCS shape. Both, the spatial dependence of $\rho(E, x)$ ( $x$ is the transport direction) and the size of the minigap can be described using a one-dimensional diffusive model where the sample geometry is approximated by two normal metal-superconductor (NS) structures weakly coupled to each other via an opaque tunnel barrier ("hard wall") such that $\rho\left(E, x=L_{\mathrm{N}}\right)$ at the tunnel barrier can be calculated neglecting the presence of the second NS structure. $\rho(E, x)$ is determined by solving the Usadel equation for the retarded Green's functions $G(E, x)$ and $F(E, x)$ numerically [17]. The DOS is given by $\rho(E, x)=\rho_{0} \operatorname{Re} G(E, x)$ with the normal-state DOS $\rho_{0}$. The length of the superconductor is assumed to be infinite, the length of the normal metal is $L_{\mathrm{N}}$, i.e. the bridge is broken symmetrically in the middle of the normal region. A second important parameter in the model is the "mismatch parameter" $\Gamma=\sigma_{\mathrm{N}} / \sigma_{\mathrm{S}}\left(D_{\mathrm{S}} / D_{\mathrm{N}}\right)^{1 / 2}$ with the conductivities $\sigma_{\mathrm{N}(S)}$ and the electronic diffusion constants $D_{\mathrm{N}(S)}$

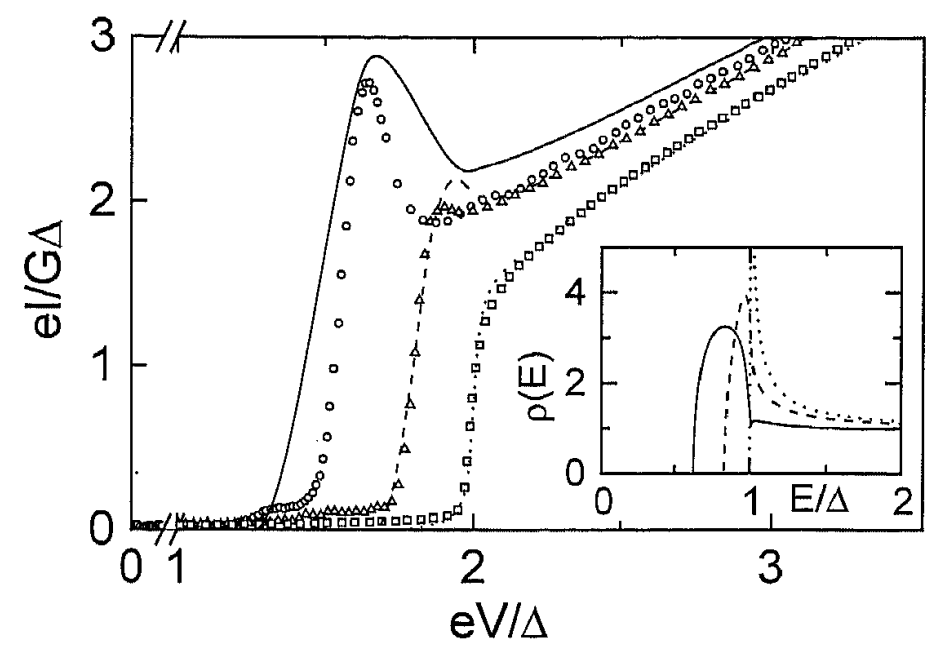

Fig. 3. Measured $I V \mathrm{~s}$ (symbols) for the Al-Au-Al samples No. 1 (triangles), No. 2 (circles) and an $\mathrm{Al}$ sample (squares) when in tunnel regime. Also shown are the calculated $I V$ of a BCS superconductor at $\Theta \leq 50 \mathrm{mK}$ (dotted line) and fittings to the $I V \mathrm{~s}$ of samples No. 1 (dashed) and No. 2 (solid) according to Ref. [17]. Inset: DOS for a BCS superconductor (dotted) and samples No. 1 (dashed) and No. 2 (solid) 
of the normal metal (superconductor). Both parameters can be estimated experimentally by the sample geometry and the RRR.

The tunnel $I V$ s are calculated by autoconvoluting the DOS. The calculated $\rho\left(E, L_{\mathrm{N}}\right)$ and $I V \mathrm{~s}$ using those parameters are displayed in Fig. 3.

When the two electrodes are brought back into contact, the IVs show the subgap-structure due to MAR [12,13]. Fig. 4 displays several $I V$ s (symbols) of single-atom contacts of samples No. 1 (left) and No. 2 (right). $G$ of all examples are smaller than or comparable to the conductance quantum $G_{0}=$ $2 e^{2} / h$. For small $G$ the maximum in the $I V$ at $2 E_{\mathrm{g}}$ is still observable and strong features appear at the sub-multiples $2 E_{\mathrm{g}} / m e(m=2,3, \ldots)$.

In order to describe the $I V \mathrm{~s}$ quantitatively we generalize the theory of MAR by introducing the Andreev-reflection amplitude $A\left(E, L_{\mathrm{N}}\right)$ calculated for our model geometry via

$$
A\left(E, L_{\mathrm{N}}\right)=-i \frac{F\left(E, L_{\mathrm{N}}\right)}{1+G\left(E, L_{\mathrm{N}}\right)}
$$

into the scattering formalism of Ref. [12] and calculate the $I V$ s for arbitrary transmission. This procedure implies that the $\mathrm{PE}$ inside one bank of the point contact is not altered when bringing the two banks into contact.

The lines in Fig. 4 are calculated within this model for a single channel with the transmission given in the figure caption. Note, that the calculation contains no free parameter since the transmission is given by the slope of the $I V$ far above the gap.

Contrary to the observations for $\mathrm{Al}$ and other superconductors $[9,15]$, we find for Au stable configurations in the contact regime whose transport properties can be explained by one single conduction channel. The transmission of this channel can be varied within a wide range of values $(0.15<T<0.98)$ by rearranging the geometry of the central region (opening and closing again the contact).
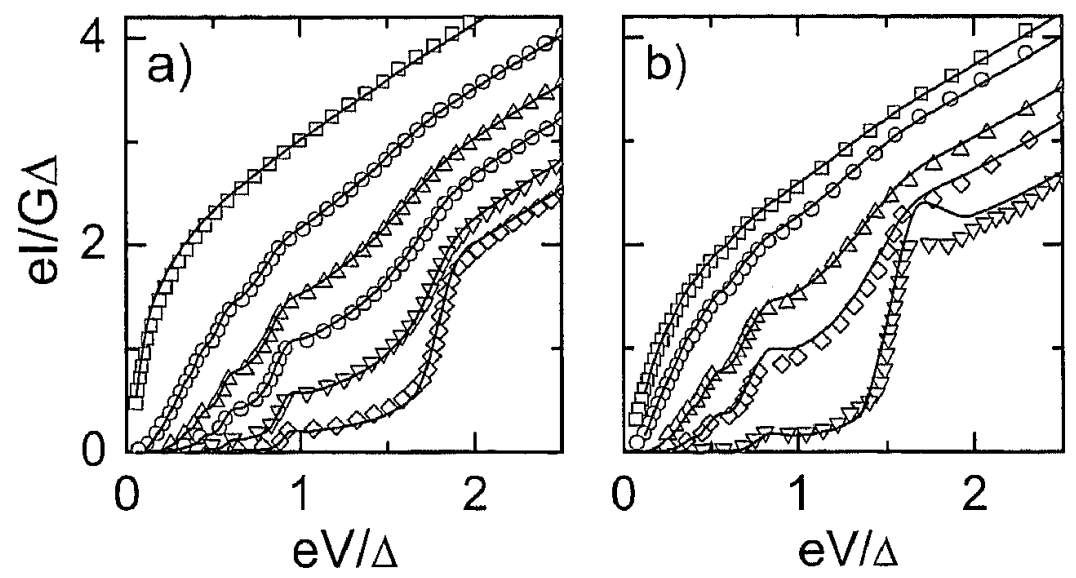

Fig. 4. a) [b)]: measured $I V$ s (symbols) of six [five] contacts of sample No. 1 [No. 2] and best numerical fits for PE superconductors (lines) with the same parameters as in Fig. 3. The transmissions are from top to bottom: $T=0.98,0.85,0.69,0.55,0.35$, and $0.15[T=0.93,0.86,0.68,0.50$, and 0.11$]$ 
Several authors have predicted within different approaches $[8,20]$ that single-atom contacts of monovalent metals as, e.g. noble metals, should transmit electrons through one single channel only. The mechanism which gives rise to a single-channel transport in Ref. [8] is the formation of a resonance of the $6 s$ band at the Fermi energy. The transmission of this channel is a function of the contact geometry. Transmission close to one is predicted only if the central atom is coupled equally to both electrodes, i.e. symmetric geometrical configuration. Disorder within several atomic layers from the central atom reduces the value of the transmission coefficient.

When further closing the contact to higher conductances a decomposition analysis as for the $\mathrm{Al}$ sample taking into account more channels has to be performed. When the closing is stopped at conductances around $2 G_{0}$, stable reproducible configurations can be achieved, which allow for repeated breaking and closing of the contact with the same conduction properties. As an example we plot in Fig. 5 a representative subset of conductance vs. distance curves of an Al-Au-Al sample, measured below $100 \mathrm{mK}$ during two days when changing the configurations. A magnetic field of $B=10 \mathrm{mT}$ has been applied to produce linear $I V \mathrm{~s}$. The data shown as solid lines have been measured when continuously opening at a speed of $10-20 \mathrm{pm} / \mathrm{s}$. For determining the channel ensemble (see lower panel of Fig. 5) two opening and closing cycles have been performed with stopping the motion at different positions and recording $I V$ characteristics. The resulting $T_{\mathrm{n}}$ are plotted in the lower panel of Fig. 5 and their sum is additionally plotted as symbols in the upper panel.

Starting at $G \approx 2 G_{0}$ from a three channel configuration with one well transmitted channel $T_{1} \approx 0.8$ and two almost degenerate ones $T_{2} \approx T_{3} \approx 0.5$, the conductance jumps down to a short and tilted plateau at around $1.2 G_{0}$, before a long last plateau with a reproducible substructure evolves. The sawtooth-like part of the plateau can be decomposed into the contributions of one widely open channel (which reveals the sawtooth behavior) and a smaller second one. The last part of the plateau has a conductance around $0.8 G_{0}$ and can be described by a single conductance channel. After breaking, the contact is closed by an almost exponential increase of the conductance to about $G \approx 0.07 G_{0}$ and a sudden jump to $G \approx 0.7 G_{0}$, still with a single channel. After closing the contact further by about $0.1 \mathrm{~nm}$, the contact jumps to one of two two-channel configurations (with different transmissions than the two-channel situation when opening). These configurations appear to be metastable since the contact remains in those states for different length intervals, which differ from repetition to repetition. The analysis of the channel ensemble reveals that one of the channels has the same transmission $T_{1} \approx 0.6$ for both configurations while the second jumps between two very different values (0.3 and 0.8 ). A possible interpretation of this behavior would be one atom in a stable position and a second one alternating between two almost degenerate positions. 


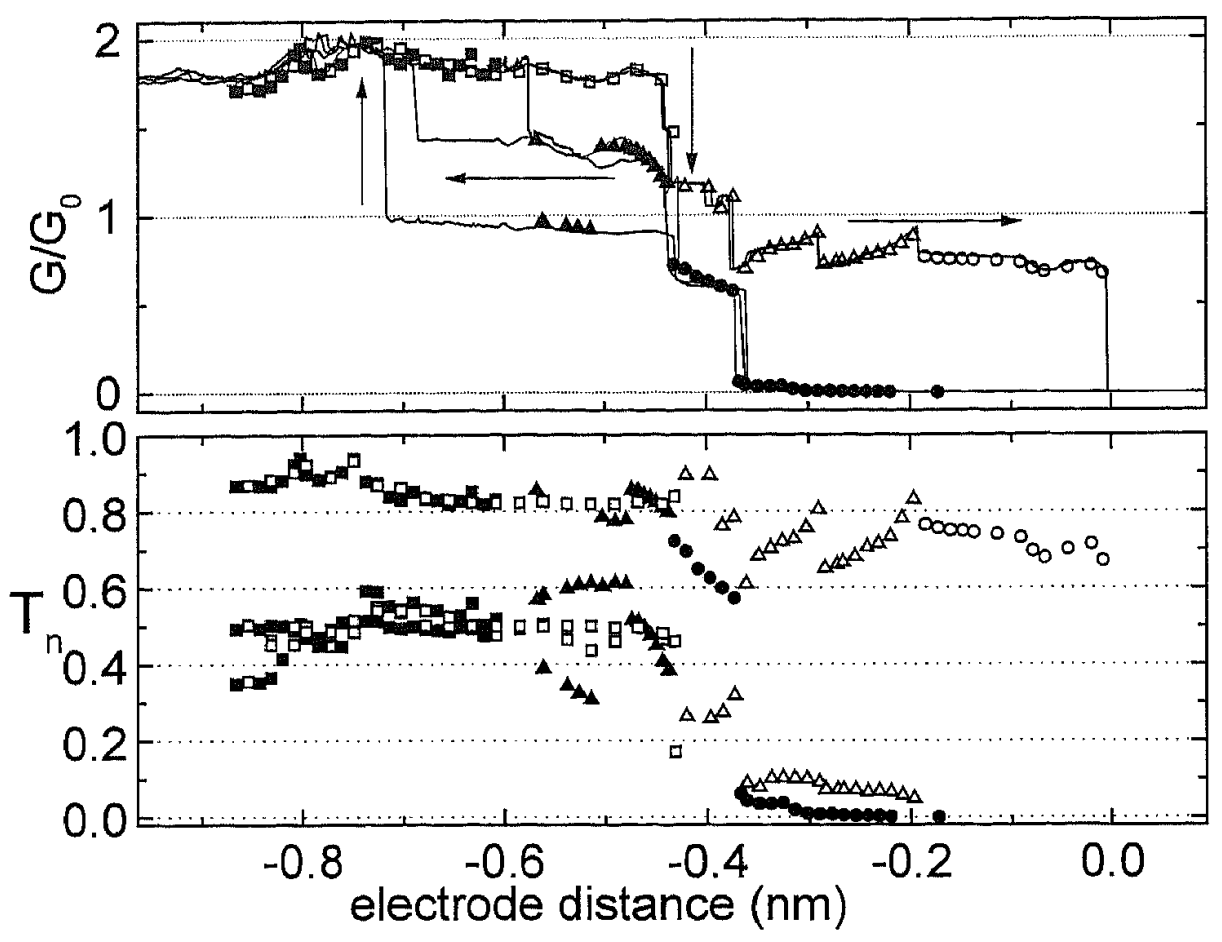

Fig. 5. Upper panel: Conductance as a function of electrode distance of Al-AuAl sample No. 3 (with $E_{\mathrm{g}} / e=125 \mu \mathrm{V}$ ) when repeatedly opened and closed. The distance axis has been scaled by the exponential dependence of $G$ in the tunnel regime. Due to hysteresis in the mechanical setup, an varying offset of about $0.1 \mathrm{~nm}$ has been subtracted such that the origin of the distance axis is for all repetitions at the position where the contact breaks. Lines: continuous motion at $v \approx 10-$ $20 \mathrm{pm} / \mathrm{s}$ and dc-conductance measurement with applied magnetic field of $10 \mathrm{mT}$ for suppressing the superconductivity, symbols: sums of the transmission values when motion was stopped for recording $I V \mathrm{~s}$ and determining the channel ensemble: Open symbols: opening, closed symbols: closing; circles (triangles, squares): one (two,three) channels. Not all data are shown. Lower panel: Transmission ensemble for opening (open symbols) and closing (closed symbols)

Finally the contact arrives at its initial stable plateau with three channels. The transmission values as well as the substructure of the plateau are reproducible within the accuracy of the conductance measurement in the continuous measurements and the determination of the channels $(\approx 3 \%)$. Although not all details of the substructure are observed in all of the $\approx 20$ repetitions', the substructure of this stable plateau can be used to distinguish the extrinsic hysteresis of the mechanical setup and the intrinsic hysteresis of the atomic motion. From the distance values we have subtracted varying offsets of the order of $0.1 \mathrm{~nm}$ such that the contacts break at zero. The mechanical hysteresis has been determined such that the curvatures of the stable three-channel plateaus when opening and closing the contact overlay. From the agreement between subsequent conductance curves we estimate the relative accuracy of the distance axis to be about $2 \%$, the absolute scaling, however, is much less accurate: $20 \%$. The remaining hysteresis between open- 


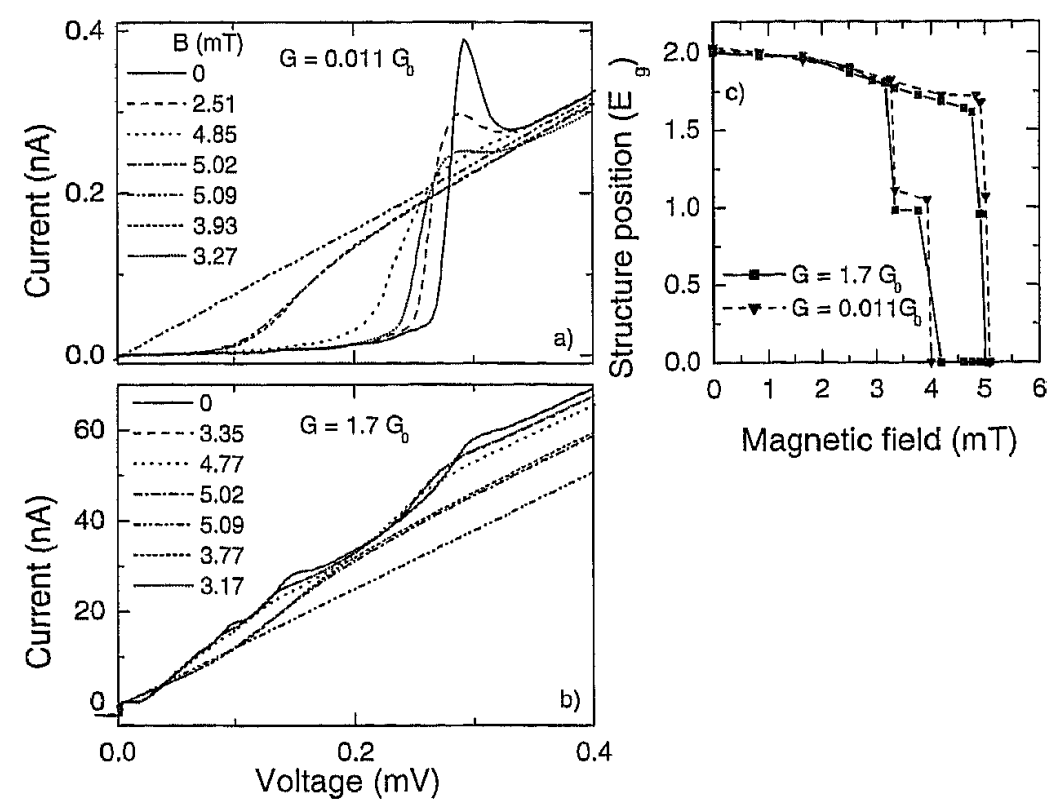

Fig. 6. a) and b) $I V$ characteristics of two different configurations of sample No. 2 at $50 \mathrm{mK}$ with applied perpendicular magnetic field. The field size has been varied according to the values given in the legend from top to bottom. The $I V \mathrm{~s}$ of panel a) [b)] have been recorded for in tunnel [contact] regime with $G=0.01 G_{0}[G=$ $\left.1.7 G_{0}\right]$. Panel c) shows the position of the maximum of $d I / d V$ in units of the minigap $E_{g}=160 \mu \mathrm{eV}$ at zero field as a function of the applied field. For a linear $I V$ the voltage of the maximum was set to zero

ing and closing of $\approx 0.4 \mathrm{~nm}$ is of the order of the lattice spacing of gold and is thus an intrinsic effect of the atomic contact [24]. The reproducible situation was kept until the contact was closed thoroughly. These observations support the assumption that the conductance of the whole system is determined by the central atom and the coupling to its nearest neighbors.

Figs. 6 a) and b) show the evolution of the subgap structure with applied magnetic field for two different contacts of sample No. 2, one in the tunnelling regime, the other in the contact regime. In our experiment the field is applied perpendicular to the film plane. As the field size is increased, the excess current is suppressed, the current steps are slightly rounded and the peak positions are gradually shifted to lower voltages. For fields larger than $5.02 \mathrm{mT}$ no submultiple current steps are observable and the apparent gap size measured by the position of the maximum of the derivative of the $I V$ is reduced to almost half of its value at zero magnetic field.

This position is plotted in Fig. $6 \mathrm{c}$ ) as a function of the applied field. When a field of $5.09 \mathrm{mT}$ or more is reached, the $I V$ becomes linear with a slope corresponding to the sum of the transmissions determined in zero field. When lowering the field down to $\approx 4.0 \mathrm{mT}$, the $I V$ remains linear. When further reducing the field, the $I V$ suddenly switches to the intermediate behavior with half gap width as observed when raising the field. For fields smaller than $\approx 3.2 \mathrm{mT}$ the observation of multiple Andreev reflections is recovered. 
When reversing the field direction the same $I V$ is observed for the same absolute value of the field, which proves that there is no residual field along the field axis. Effects of the earth magnetic field or spurious fields in different directions however cannot be excluded. A similar behavior is observed for contacts of sample No. 1 but with slightly higher absolute values of the critical fields. E.g. the $I V$ becomes linear for fields above $6.35 \mathrm{mT}$, switches back to the intermediate state at $5.3 \mathrm{mT}$ and to the fully superconducting state at $4.4 \mathrm{mT}$.

For explaining these observations we summarize briefly the behavior of a proximity superconductor in an external magnetic field (see e.g. [25] and references therein). A characteristic feature of proximity effect is the appearance of a so-called breakdown field instead of a critical field for superconductors at which the external field starts to penetrate the sample due to the reduced screening capability of the proximity system. Below a certain temperature $T^{\star}$, determined by the dimension and the effective penetration depth $\lambda_{\mathrm{N}}$ of the normal layer, the transition to the normal state becomes of 1st order, like for Type I superconductors. I.e. a "supercooling" and a "superheating" of the proximity superconducting state becomes possible between two limiting field scales $H_{\mathrm{s} h} \approx \Phi_{0} / \lambda_{\mathrm{N}}^{2}$ (superheating field) and $H_{\mathrm{sc}} \approx \Phi_{0} / \xi\left(L_{\mathrm{N}}\right)^{2}$, the supercooling field, where $\xi\left(L_{\mathrm{N}}\right)$ describes an effective coherence length. The exact values of $H_{\mathrm{sc}}$ and $H_{\mathrm{s} h}$ depend on the degree of disorder of the electronic transport in the normal conductor, the NS interface and the sample dimensions.

Important for the discussion here is the fact that $H_{\mathrm{sc}}$ and $H_{\mathrm{s} h}$ depend differently on the strength of the PE in both electrodes. The important physical quantity is the magnetic penetrating the two electrodes. For simplicity, we model the difference in the PE at the point where the junction breaks by different dimensions of the normal metal. Since we do not control on the atomic level where the junction breaks it is likely that the bridge does not break exactly in the middle of the normal part, but that two parts with slightly different lengths $L_{\mathrm{N} 1}$ and $L_{\mathrm{N} 2}=2 L_{\mathrm{N}}-L_{\mathrm{N} 1}$ are formed. For $L_{\mathrm{N} 1} \neq L_{\mathrm{N} 2}$ the characteristic field scales will be different for both bridge arms. A quantitative description of the influence of the magnetic field is difficult because of the complicated shape of the samples. However, we can assume that the point contact spectra are mainly determined by the superconducting properties at the constriction where the voltage drops, i.e. depend on the length scales $L_{\mathrm{N} 1}$ and $L_{\mathrm{N} 2}$.

With these approximations we interpret our observations as follows (see Fig. 7): With increasing magnetic field the structures in the $I V$ are slightly rounded and shifted to lower voltages since the screening is reduced due to the finite elastic mean free path. However this effect is much smaller than observed for pure Al samples [23]. When the superheating field $H_{s h 2}$ of the longer bridge arm with length $L_{N 2}$ is reached, this part becomes normal, giving rise to an $I V$ characteristic of a NS-junction, i.e. with a current onset 


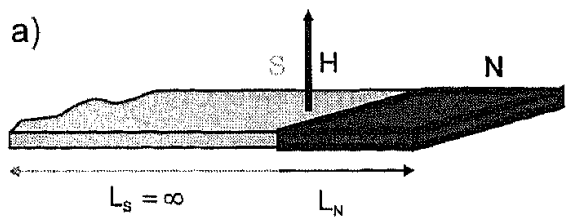

b)

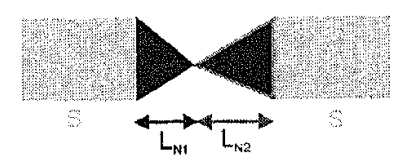

c)

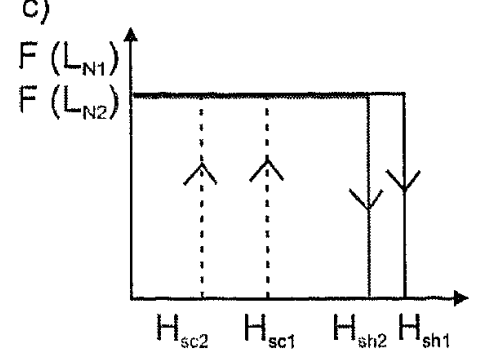

d)

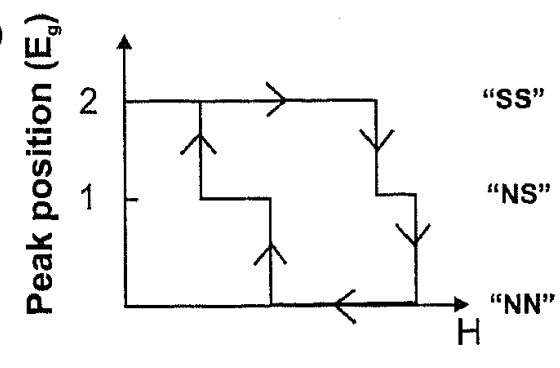

Fig. 7. Panel a) displays the model geometry of one bridge arm used for calculating the superconducting properties at the end of the normal metal where it makes contact with the other bridge arm. Panel b) represents schematically the sample geometry when the bridge is not broken exactly in the middle of the normal region. In panel c) we show schematically the development of the pair amplitudes at the constriction for both bridge arms as a function of the applied field and panel d) the resulting effective behavior of the samples in the different field ranges

at $e V \approx E_{g}$ and without MAR features. When further increasing the field, the superconductivity is completely suppressed when $H_{s h 1}$ of the shorter bridge arm is exceeded, i.e. the $I V$ becomes linear. When reducing the field, both bridge arms remain normal conducting until the supercooling field $H_{s c 1}$ of the shorter bridge arm is reached. Below this field this bridge arm becomes superconducting again, giving rise to an NS-like $I V$. Only below $H_{s c 2}$ of the longer bridge arm the field is expelled from both parts and the $I V$ with MAR structures is recovered. At zero external field the decomposition of the $I V$ into the contribution of individual channels yields the same channel ensemble es before applying the field.

As already mentioned, a quantitative description of this behavior would require a more realistic modelling of the sample geometry. A rough estimate shows that a length difference of the bridge arms in the order of $10 \%$ to $20 \%$ of $L_{N}$ would account for the observed difference in the field scales. For determining the density of states and Andreev reflection amplitudes in zero field (used for calculating the $I V \mathrm{~s}$ in the contact regime) we assumed a symmetrically broken bridge. However, a length difference of the order of $20 \%$ would only slightly affect those results and has therefore been neglected.

To summarize, we have presented an investigation of the transport properties of gold tunnel and few-atom contacts having superconducting leads. The current-voltage characteristics in the tunnel regime strongly differ from what is observed for a pure BCS superconductor. From their analysis within the framework of the diffusive proximity effect, we extracted the quasiparticle density of states at the contact region and the corresponding Andreev reflection amplitude. This last ingredient was then used to extend the theory of 
MAR to the case of a channel with arbitrary transmission between PE superconductors. Although this extended model does not account quantitatively for all the details of the $I V \mathrm{~s}$ of few-atom contacts, it does explain the general trends. An important conclusion of this analysis is that the smallest gold contacts observable in the experiments accommodate a single conduction channel in accordance with theoretical predictions for single-atom contacts. The PE strongly influences the behavior in a perpendicular magnetic field where a hysteretic transition to the normal state is observed. We have demonstrated here, that it is possible to drive a particular contact reproducibly into the normal state and back to the superconducting state without changing $\left\{T_{\mathrm{n}}\right\}$. We stress the high stability of the setup necessary for maintaining a particular contact stable during the measurement series.

We thank C. Bruder, M.H. Devoret, D. Esteve, J. M. van Ruitenbeek, G. Schön and C. Strunk for helpful discussions. We have enjoyed fruitful interaction with D. Averin, J. C. Cuevas and A. Levy Yeyati, and we thank them for providing us with their respective computer codes. This work was partially supported by the BNM (France) and the DFG (Germany).

\section{References}

1. G. Binnig et al., Appl. Phys. Lett. 40, 178 (1982).

2. M.F. Crommie et al., Science 262, 218 (1993).

3. G. Binnig and H. Rohrer, IBM J. Res. Dev. 30, 355 (1986).

4. N.D. Lang, Phys. Rev. B 52, 5335 (1995); C.C. Wan et al., Appl. Phys. Lett. 71, 419 (1997); A. Yazdani et al., Science 272, 1921 (1996).

5. R. Landauer, Philos. M. 21, 863 (1970).

6. B. Ludoph and J.M. van Ruitenbeek, Phys. Rev. B 59, 12290 (1999); H.E. van den Brom and J.M. van Ruitenbeek, Phys. Rev. Lett. 82, 1526 (1999); B. Ludoph et al., ibid. 82, 1530 (1999); M.F. Goffman et al., ibid. 85, 170 (2000); R. Cron et al., ibid. 86, 4104 (2001).

7. J.C. Cuevas et al., Phys. Rev. Lett. 80, 1066 (1998).

8. A. Levy Yeyati et al., Phys. Rev. B 56, 10369 (1997).

9. E. Scheer et al., Phys. Rev. Lett. 78, 3535 (1997)

10. M. Octavio et al., Phys. Rev. B 27, 6739 (1983).

11. G.B. Arnold, Journal of Low Temp. Phys. 68, 1 (1987).

12. D. Averin and A. Bardas, Phys. Rev. Lett. 75, 1831 (1995).

13. J.C. Cuevas et al., Phys. Rev. B 54, 7366 (1996).

14. E.N. Bratus et al., Phys. Rev. B 55, 12666 (1997).

15. E. Scheer et al., Nature 394, 154 (1998).

16. D. Esteve in Mesoscopic Electron Transport, L.L. Sohn et al.(Eds.) (Kluwer, Dordrecht, 1997) pp. 375 and references therein.

17. W. Belzig et al., Superlattices and Microstructures 25, 1251 (1999).

18. L. Olesen et al., Phys. Rev. Lett. 72, 2251 (1994).

19. N. Agraït et al., Phys. Rev. B 47, 12345 (1996).

20. J.M. van Ruitenbeek in Mesoscopic Electron Transport, L.L. Sohn et al. (Eds.) (Kluwer, Dordrecht, 1997) pp. 549 and references therein.

21. J.M. van Ruitenbeek et al., Rev. Sci. Inst. 67, 108 (1996). 
22. E. Scheer et al., Phys. Rev. Lett. 86, 284 (2001).

23. E. Scheer et al., Physica B 280, 425 (2000).

24. C. Untiedt et al., Phys. Rev. B 56, 1251 (1997).

25. A.D. Zaikin, Solid State Commun. 41, 533 (1982); W. Belzig et al., Phys. Rev. B 53, 5727 (1996); A.L. Fauchère and G. Blatter, ibid. 56, 14102 (1997); W. Belzig et al., ibid. 58, 14531 (1998). 